\title{
DNA demethylation enhances myoblasts hypertrophy during the late phase of myogenesis activating the IGF-I pathway
}

\author{
Pamela Senesi • Livio Luzi • Anna Montesano • \\ Ileana Terruzzi
}

Received: 8 February 2013 / Accepted: 27 November 2013/Published online: 24 December 2013

(C) The Author(s) 2013. This article is published with open access at Springerlink.com

\begin{abstract}
Skeletal muscle regeneration and hypertrophy are important adaptive responses to both physical activity and pathological stimuli. This research was performed to investigate DNA demethylation action on the late phase of muscle differentiation and early stage of hypertrophy. The epigenetic process involved in myogenesis was studied with the DNA-demethylating agent 5-azacytidine (AZA). We induced muscle differentiation in $\mathrm{C} 2 \mathrm{C} 12$ mouse myoblasts in the presence of $5 \mu \mathrm{M}$ AZA and growth or differentiation medium for 48,72 , and $96 \mathrm{~h}$. To study a potential AZA hypertrophic effect, we stimulated $72 \mathrm{~h}$ differentiated myotubes with AZA for $24 \mathrm{~h}$. Unstimulated cells were used as control. By western blot and immunofluorescence analysis, we examined AZA action on myogenic regulatory factors expression, hypertrophic signaling pathway and myotube morphology. During differentiation, protein levels of myogenic markers, Myf6 and Myosin Heavy Chain (MyHC), were higher in AZA stimulated cells compared to control. Myostatin and p21 analysis revealed morphological changes which reflect a tendency to hypertrophy in myotubes. In AZA stimulated neo formed myotubes, we observed that IGF-I pathway, kinases
\end{abstract}

P. Senesi - L. Luzi · A. Montesano

Department of Biomedical Sciences for Health, University

of Milan, Milan, Italy

P. Senesi · L. Luzi

Metabolism Research Centre and Department of Endocrinology and Metabolic Diseases, San Donato Hospital and Scientific Institute, Milan, Italy

\section{Terruzzi $(\bowtie)$}

Division of Metabolic and Cardiovascular Science, Metabolism, Nutrigenomics and Cellular Differentiation Unit, San Raffaele Scientific Institute, Milan, Italy

e-mail: terruzzi.ileana@hsr.it p70 S6, 4E-BP1, and ERK1/2 were activated. Furthermore, AZA treatment increased MyHC protein content in stimulated neo myotubes. Our work demonstrates that DNA demethylation could plays an important role in promoting the late phase of myogenesis, activating endocellular pathways involved in protein increment and stimulating the hypertrophic process.

Keywords Muscle differentiation - DNA methylation 5-Azacytidine $\cdot$ Hypertrophic process $\cdot \mathrm{C} 2 \mathrm{C} 12$ myoblasts · IGF-I

\section{Introduction}

Skeletal muscle regeneration and hypertrophy are important adaptive responses to both disease and physical activity [1]. Muscle repair is controlled by satellite cells (SC), representing skeletal muscle stem cells [2]. In response to injury, quiescent SC start to proliferate generating myogenic precursor cells (myoblasts). Myoblasts withdraw from the cell cycle and form myotubes [3].

This process is principally governed by muscle regulatory factors (MRFs), including MyoD and Myf6 [4, 5]. MRFs regulate the cellular cycle progression, in particular Myf6 cooperates with $\mathrm{p} 21$, a cell cycle regulator protein, to promote muscle specific protein synthesis [6] such as Myosin Heavy Chain (MyHC). Instead, the end of myogenesis [7] is lead by Myostatin (Mnst). Mstn, a member of the transforming growth factor- $\beta$ superfamily, is an important negative regulator of skeletal muscle development. Naturally, Mnst occurring mutations, as well as experimental knockout of the Mstn gene, lead to hypermuscular phenotype [8]. In vitro model of skeletal muscle cells, Mstn is predominantly localized in the nuclei of 
differentiated, polynucleated myotubes and down regulates the muscle genes expression [9].

Muscle hypertrophy is an increment of existing muscle fibers size [10], associated with an enhanced protein accumulation. Insulin growth factor I (IGF-I) are crucially involved in hypertrophic process induced by Growth Hormone $(\mathrm{GH})$ treatment or exercise [11, 12]. In recent years, Spangerburg and other investigators have tried to understand the mechanisms by which IGF-I may mediate changes in muscle mass during mechanical loading. [1315]. The data, obtained by these researchers, are in part controversial [16-19]: inhibition of IGF-I activity does not eliminate increased muscle mass through mechanical load, but driving IGF-I enhances the effects of load. Although a controversial point of view, there is no doubt that IGF-I could play a central role in muscle hypertrophy and adaptation. In fact, IGF-I overexpression is sufficient to induce muscle hypertrophy, modulating the entire circuit necessary to guarantee it: an increase in protein synthesis, a decrease in protein degradation, an activation and a fusion of satellite cells [20-22].

Insulin growth factor I binding activates the IGF-I receptor (IGF-I R), a receptor tyrosine kinase. The IGF-I R subsequently recruits the insulin receptor substrate (IRS-1), which results in the activation of two signaling pathways: the mitogen-activated protein kinases (MAPK) pathway and the phosphatidylinositol 3-kinase (PI-3 K) pathway $[23,24]$.

The MAPK pathway is crucial in mitosis-competent cells for cell proliferation and survival [25]. Extracellular signal regulated kinases (ERK1 and ERK2), members of the MAPK family, are involved in the regulation of muscle mass. Myoblasts/myotubes have a unique biphasic requirement for ERK activity [26]. ERK1/2 are critical for growth factor-induced cellular proliferation, inhibitory to myoblastic differentiation. These kinases are required for myotube fusion and appear critical to this last process [27]. Moreover, in adult skeletal muscle, high-intensity exercises have been shown to activate the MAPK-ERK pathway and in vivo studies showed that MAPK-dependent pathways affect both fiber size and fiber type [28].

The PI-3 K pathway is the predominant pathway that stimulates muscle protein synthesis and is believed to be required for a normal hypertrophic response. PI-3 K direct target is AKT [10, 24]. Under normal conditions, AKT activation results in the formation of a signaling complex termed TORC1, an important component of which is mTOR [29]. Activation of mTOR leads to phosphorylation of ribosomal protein S6 Kinase (p70 S6 K). p70 S6 K phosphorylates an important ribosomal subunit that is necessary for muscle protein translation, and deletion of p70 S6 K in muscle results in smaller muscle fibers [30].
Furthermore, mTOR directly phosphorylates the eukaryotic initiation factor 4E (4E-BP1). Once phosphorylated, 4E$\mathrm{BP} 1$ releases its inhibitory effect on the translation initiation factor elF4E, which impairs inhibition of translation initiation by coupling with the end CAP of mRNA [30, 32].

Recently, discoveries in the field of skeletal muscle biology have made an effort to understand how epigenetic modifications affect the myogenic lineage acquisition [33].

The observation that treatment with a methyltransferase inhibitor, 5-azacytidine (AZA), converts C3H10T1/2 embryonic fibroblasts into muscle cells providing the first relationship between DNA methylation and myogenesis [34]. This correlation is further underscored by the finding that promoters of MRFs, MyoD and Myogenin, are demethylated during muscle cell differentiation [35, 36]. However, the specific impact of DNA demethylation on late phase of differentiation and on muscle mass regulation is not completely understood.

In our study, we used AZA to induce DNA demethylation during differentiation stages of $\mathrm{C} 2 \mathrm{C} 12$ cell line, an established model of satellite cell growth and differentiation $[37,38]$. Our data show that DNA demethylation could stimulate myoblasts differentiation and promote hypertrophic process, through the activation of IGF-I pathway.

\section{Materials and methods}

\section{Materials}

Anti calnexin (H-70), anti MyoD (C-20), anti Myf6 (C-19), anti Myostatin (GDF-8: N-19), anti MyHC (H-300), anti p21 (C-19), anti IGF-I receptor- $\beta$ (C-20), anti phosho p70 S6-kinase (Thr421/Ser 424), anti p70 S6-Kinase (C-18), anti 4E-BP1 (R-113), anti eIF-4E (P-2), anti p-ERK1/2 (E-4), anti-ERK1 (K-23), and anti ERK2 (C-14) primary antibodies and peroxidase or rhodamine-conjugated secondary antibodies were purchased from Santa Cruz Biotechnology (Santa Cruz, CA, USA). All other reagents were purchased from Sigma Chem. Co. (St. Louis, MO, USA). Mouse C2C12 myoblastic cells were purchased from European Collection of Animal Cell Cultures (ECACC).

\section{Experimental protocol}

$\mathrm{C} 2 \mathrm{C} 12$ myoblasts were cultured at $37^{\circ} \mathrm{C}$ in humidified $5 \% \mathrm{CO}_{2}$ atmosphere in growth medium (GM), containing DMEM (Dulbecco Modified Eagle Medium) supplemented with $20 \%$ (v/v) FBS (fetal bovine serum), $1 \%$ penicillinstreptomycin, and $1 \%$ L-glutamine. Differentiation process is initiated upon reaching subconfluence by switching C2C12 myoblasts in differentiation medium (DM), 
containing DMEM supplemented with $1 \%$ horse serum, antibiotics, and $1 \%$ L-glutamine [38]. While, $\mathrm{C} 2 \mathrm{C} 12$ cells, maintained in GM, spontaneously fuse in neo myotubes as a result of the achievement of myoblasts confluence [39].

To study DNA demethylation action on differentiation process, cells, seeded at $6 \times 10^{2}$ cells $/ \mathrm{cm}^{2}$, were grown until $80 \%$ of confluence and then were maintained in GM or DM containing $5 \mu \mathrm{M}$ AZA (GMAZA and DMAZA). $5 \mu \mathrm{M}$ AZA represents the optimal concentration to stimulate cells without cytotoxic effects [34, 40]. This evidence was, furthermore, validated by our dose response experiments (data not shown). Control cells were maintained in GM or DM. Medium, with or without AZA, was changed every $24 \mathrm{~h}$. Cells were analyzed during intermediate $(48 \mathrm{~h})$ and late (72 and $96 \mathrm{~h}$ ) differentiation phases.

To investigate DNA demethylation effects on hypertrophic process, we used $\mathrm{C} 2 \mathrm{C} 12$ cells after $72 \mathrm{~h}$ from differentiation induction in DM: in our model, most of the myotubes were completely formed in this phase. Neo formed myotubes were treated with $5 \mu \mathrm{M}$ AZA for $24 \mathrm{~h}$ (AZAMT). Unstimulated cells (DMMT) were used as control.

Growth curve and cell vitality test

C2C12 myoblasts were plated in $35 \mathrm{~mm}^{2}$ culture dishes and grown in presence of GMAZA or DM. Control cells were cultured in GM. Experiment continued until the control cells have reached subconfluence [41]. Every day, the cells were trypsinized and stained with trypan blue. Both viable (non-stained) and non-viable (blue) cells were counted using a hemacytometer. The total cell count average values for each single day were used to plot a growth curve for treated and controlled myoblasts. Cell vitality was calculated by dividing the non-stained viable cell count by the total cell count.

\section{Global DNA methylation assay}

The total genomic DNA was extracted from the cells (treated with GM, DM, GMAZA or DMAZA) using the Qiagen DNA Mini Kit (Qiagen Sciences, Maryland, MD) following the manufacturer's instructions. The integrity and purity of DNA were spectrophotometrically examined according to its A260/A280 absorption. The Global DNA methylation levels were determined using MethylFlash ${ }^{\mathrm{TM}}$ Methylated DNA Quantification Kit (Epigentek, NY, USA) according to the manufacturer's instruction. The methylated fraction of DNA is recognized by a 5-methyl-cytosine (5-mC) antibody. With this colorimetric kit, the amount of methylated DNA, which is proportional to the optical density intensity, is quantified through an enzyme-linked immunosorbent assay-like reaction (ELISA assay). The reference DNA fragments containing 5-mC and 5-cytosine are used respectively as the positive standard and negative control to generate the standard curve. In particular, manufacturer's instruction indicates that the percentage of 5 -mC in the total DNA is calculated using the following formula:

$\% 5-\mathrm{mC}=\frac{\text { Sample OD }- \text { Negative control OD }}{2 \times \text { Slope } \times \text { Input DNA amount }} \times 100 \%$

Slope of the standard curve is determined using linear regression [42].

Immunoblotting analysis

$\mathrm{C} 2 \mathrm{C} 12$ cells were homogenized in lysis buffer $(50 \mathrm{mM}$ Tris/HCl, pH 7.4, $150 \mathrm{mM} \mathrm{NaCl}, 1 \%$ Triton X-100, $1 \mathrm{mM}$ sodium orthovanadate $\left(\mathrm{Na}_{3} \mathrm{VO}_{4}\right), 1 \mathrm{mM}$ EDTA, $1 \mathrm{mM}$ PMSF, $1 \mathrm{mg} / \mathrm{ml}$ aprotinin, $1 \mathrm{mg} / \mathrm{ml}$ leupeptin, and $1 \mathrm{mg} /$ $\mathrm{ml}$ pepstatin) and the obtained samples were sharked for $1 \mathrm{~h}$ at $4{ }^{\circ} \mathrm{C}$. Detergent-insoluble material was removed from the cell suspension by centrifugation at $12,000 \times g$ for 30 min. Protein content was quantified using Bradford method. Aliquots of $30 \mu \mathrm{g}$ supernatant proteins were resolved by SDS-PAGE and transferred to nitrocellulose membrane (Protran ${ }^{\circledR}$ Whatman ${ }^{\circledR}$ Schleicher \& Schuell), as described [43]. The membranes were incubated with specific primary antibodies and then with HRP-conjugated secondary antibodies. Equal protein loading per sample was confirmed using antibody anti-calnexin. Immunoreactive bands were visualized by an enhanced chemiluminescence method (Amersham Pharmacia Biotech, Piscataway, NJ, USA). Densitometric analysis was performed using the Scion Image Software (Scion Corporation, Frederick, MD, USA).

Immunofluorescence analysis and phase contrast microscopy

Cells were fixed in $4 \%$ paraformaldehyde, and indirect immunofluorescence analysis was performed as described [43]. Cells were observed using fluorescence Leica DM IRE2 microscopy and Nikon Eclipse 50I microscopy. The images of myotubes were captured using respectively IM50 software and Nis-Elements D 4.00 software (Leica Microsystems, Switzerland and Nikon Instruments Europe $\mathrm{BV}$, Netherlands). To verify that cells number in all conditions was superimposable, nuclei were revealed with DAPI staining. For myotube diameter and length dimensions, the average measurement was generated from approximately 150 myotubes. Ten fields were randomly chosen and all MyHC-positive multinucleated cells (at least 3 nuclei) in each field were measured. In detail, an 
a
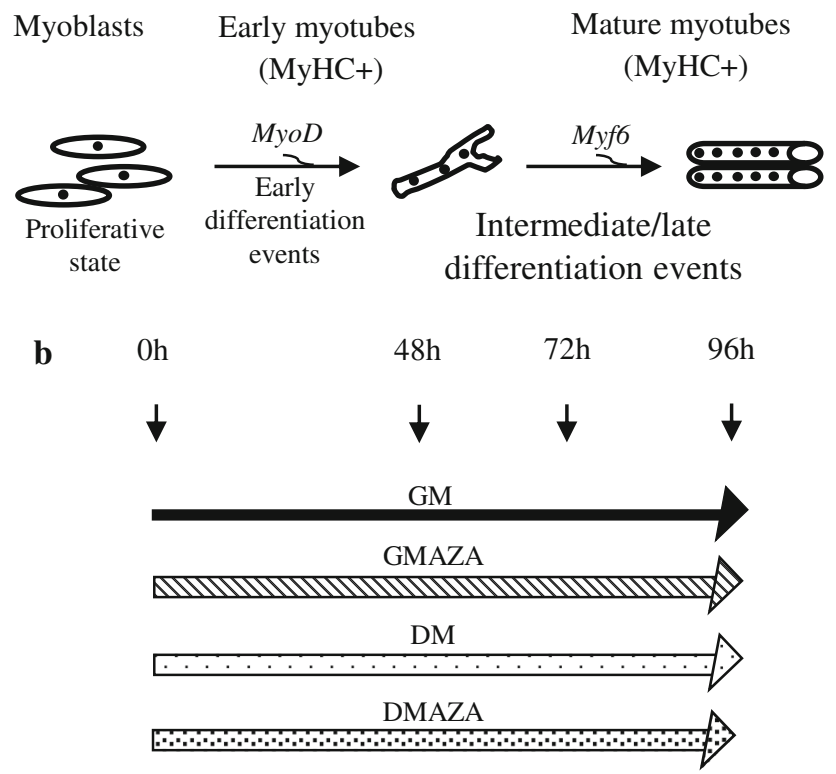

Fig. $1 U p$ schematic illustration of skeletal muscle differentiation. Down simplified design of experimental procedures

average diameter per myotube was calculated as the mean of three measurements taken along the length of the myotube. As proposed by Menconi et al. [44], the measurements were conducted in a "blinded" fashion on coded pictures with the investigator being unaware of the group from which the cultures originated. The experiments were repeated three times. Live $\mathrm{C} 2 \mathrm{C} 12$ cells were examined and images acquired by phase contrast microscopy using the same microscope and digital system described above.

Statistical analysis

Data are converted to fold change (FC) relative to the control and presented as the mean \pm SD. Statistical significances differences between treatments were analyzed using one-way ANOVA and Bonferroni post test or t-tests. Results were considered significant when $p \leq 0.05$.

\section{Results}

In our differentiation model, early myotubes appeared $48 \mathrm{~h}$ after differentiation induction (intermediate phase), and myotubes formation was completed after $72-96 \mathrm{~h}$ (late phase). We analyzed DNA demethylation effects, during these stages, adding AZA both in GM and DM cultured cells (GMAZA-DMAZA) at the beginning of the differentiation process. In the first case (GMAZA), we would delineate the possible ability of DNA demethylation to accelerate myoblast spontaneous differentiation due to cell-cell contact; and in the second (DMAZA), we would determinate the probable role of DNA hypomethylation to improve myogenesis process (Fig. 1).

First, proliferation and cell vitality assays were performed in proliferative myoblasts to verify that $5 \mu \mathrm{M}$ AZA did not have toxic effect (Fig. 2a, b). The cell growth curves showed that AZA and DM induced a significant reduction in the cell growth rate in relation to the untreated control (Fig. 2a). It is important to emphasize that AZA action did not show a cytotoxic effect in $\mathrm{C} 2 \mathrm{C} 12$ cells, as revealed cell vitality assay (Fig. 2b). Moreover, in our previous work, we in detail analyzed AZA effect on myoblasts cell cycle progression: we demonstrated that this DNA methylation inhibitor enhances myogenesis induction modulating expression of checkpoint genes involved in cell cycle progression and arrest, but no genes involved in apoptosis phenomena. In fact, GMAZA cells showed a p53 protein content comparable to that of GM cells [45].

Also, to determine whether AZA produces changes in the methylation patterns, we also studied the global DNA methylation by specifically measuring levels of 5-mC in an ELISA-like microplate-based format. As shown in Fig. 2c, we did not observe significant DNA methylation status changes in cells maintained in GM. In contrast, GMAZA and DMAZA cells were characterized by dramatic global DNA methylation reduction (At $48 \mathrm{~h}$ FC: $0.44 \pm 0.07$ GMAZA vs. GM, at $72 \mathrm{~h}$ FC: $0.35 \pm 0.08$ GMAZA vs. GM, at 96 h FC: $0.33 \pm 0.13$ GMAZA vs. GM; at 48 h FC: $0.31 \pm 0.16$ DMAZA vs. GM, at $72 \mathrm{~h}$ FC: $0.17 \pm 0.03$ DMAZA vs. GM, at 96 h FC: $0.38 \pm 0.003$ DMAZA vs. $\mathrm{GM} ; \mathrm{F}=34.35, p \leq 0.00001$ anova test followed by Bonferroni post hoc test). In according to the previous data [33, 40], we noticed a significant DNA hypomethylation status in DM condition (at $48 \mathrm{~h}$ FC: $0.42 \pm 0.09 \mathrm{DM}$ vs. $\mathrm{GM}$, at $72 \mathrm{~h}$ FC: $0.59 \pm 0.14 \mathrm{DM}$ vs. $\mathrm{GM}$, at $96 \mathrm{~h} \mathrm{FC}$ : $0.52 \pm 0.05 \mathrm{DM}$ vs. $\mathrm{GM} ; \mathrm{F}=34.35, p \leq 0.00001$ anova test followed by Bonferroni post hoc test). In detail, DNA methylation status is similar in DM and GMAZA, suggesting that AZA could improve differentiation process regulating epigenetic mechanisms in analogous manner to DM. Furthermore, exposure to DM and AZA resulted in the most significant DNA methylation decreases, indicated that AZA could strengthen muscle differentiation induced by DM. Immunostaining of $\mathrm{C} 2 \mathrm{C} 12$ cells with MyHC antibody at $48 \mathrm{~h}$ supports the DNA global methylation result: there was an evident increment in the number of MyHC-positive cells in AZA treated than untreated cells (Fig. 2d). These data confirm DNA demethylation action of AZA and indicating that epigenetics regulation is a crucial aspect of skeletal muscle differentiation.

For the success of the myogenesis program, sequential expression of MRFs, at specific step, is tightly important 
Fig. 2 AZA effects on myoblasts proliferation and on global DNA methylation status. a Growth curve trends were obtain growing myoblasts until $40 \%$ confluence in GM and then were switched in GMAZA or in DM. The experiment continued until the cells reached subconfluence. GMAZA and $\mathrm{DM}$ negative influence $\mathrm{C} 2 \mathrm{C} 12$ proliferative potential. b Myotoxicity of AZA in vitro: AZA did not induce cell death. c Evaluation of AZA demethylation action on global DNA methylation status. Global DNA methylation levels were evaluated by an ELISA assay specific for 5-mC.

d Immunofluorescence MyHC analysis at $48 \mathrm{~h}$ in $\mathrm{GM}$, GMAZA, DM, and DMAZA conditions. MyHC positive cells are labeled in red. DAPI and merge images are reported
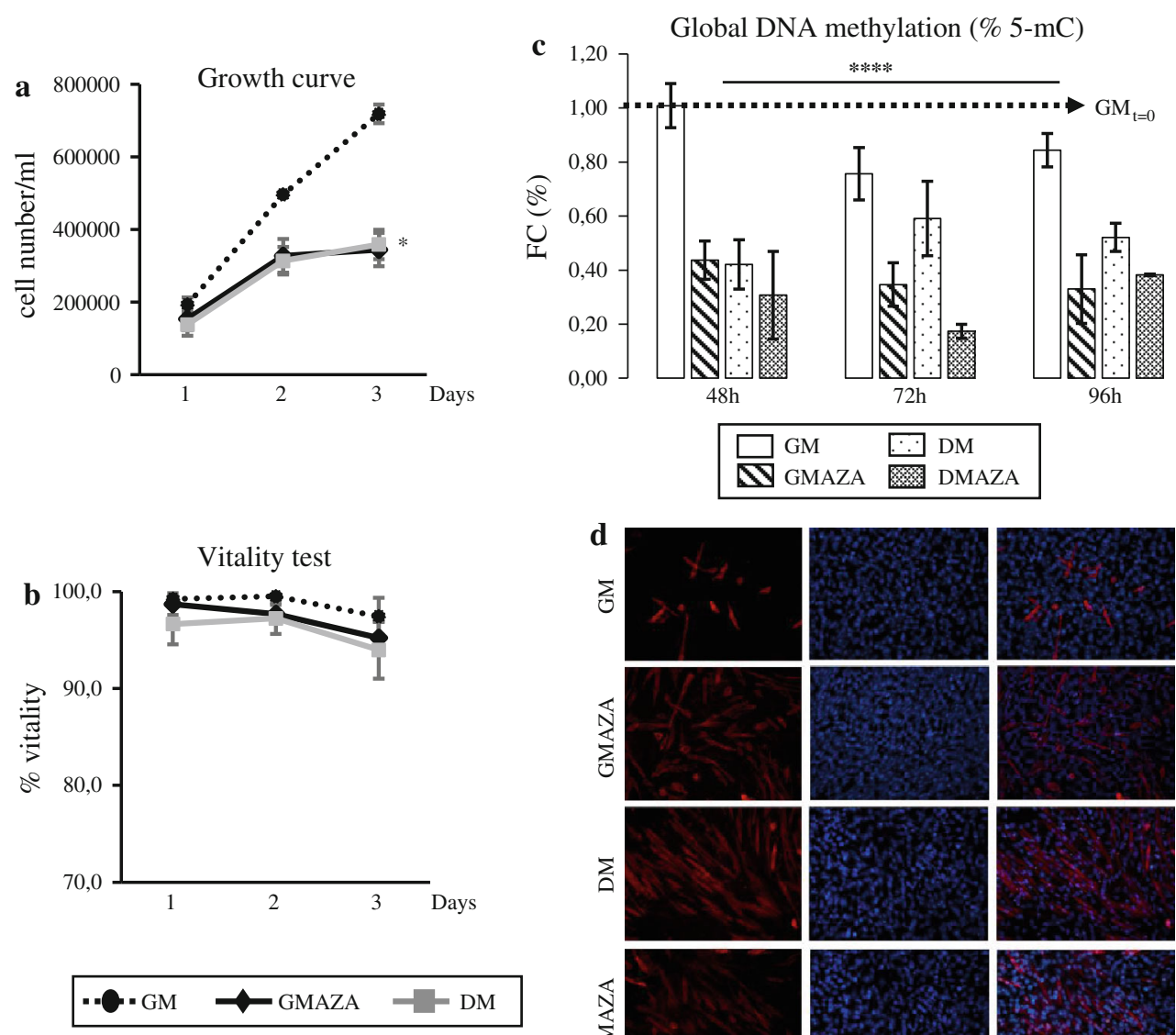
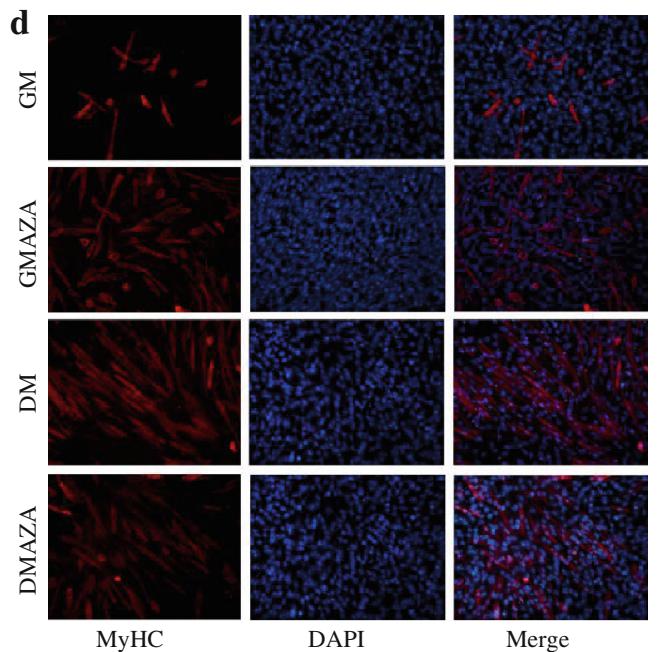

DAPI

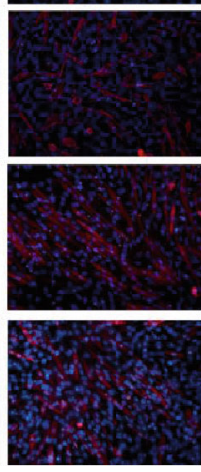

Merge
$[3,4]$. In detail, MyoD reaches the highest expression in early phase and represents a marker for this stage [5, 33]. Instead, Myf6 and skeletal protein MyHC, being expressed during intermediate and late stages of differentiation, are used as markers of these phases [33]. To characterize AZA action on differentiation progression, we performed a detailed temporal study of MyoD, Myf6, and MyHC protein synthesis (Fig. 3a).

Western blot studies showed that MyoD levels were improved (Fig. 3b, FC: $1.22 \pm 0.01 \mathrm{DM}$ vs. GM) only in DM cells during intermediate differentiation phase $(48 \mathrm{~h})$, according to the previous data $[3,46]$. At $48 \mathrm{~h}$, unchanged MyoD expression, observed in AZA treated cells, is maybe due to AZA ability to promote MyoD synthesis. Probably in AZA treated cells, MyoD peak synthesis occurred in the hours before and MyoD protein content is returned to baseline levels at $48 \mathrm{~h}$. In fact, at 72 and $96 \mathrm{~h}$, no significant differences in MyoD content was established between AZA treated and control cells.

As shown in Fig. 3c, at $48 \mathrm{~h}$ AZA considerably raise Myf6 protein content and in DMAZA cells this effect was detectable until the late stage (At $48 \mathrm{~h}$ FC: $1.66 \pm 0.03$ DMAZA vs. GM, at $72 \mathrm{~h}$ FC: $3.16 \pm 0.08$ DMAZA vs.
$\mathrm{GM}, \quad$ at $96 \mathrm{~h}$ FC: $2.89 \pm 0.09$ DMAZA vs. GM; $\mathrm{F}=19.41, p \leq 0.00001$ anova test followed by Bonferroni post hoc test). Finally, we investigated MyHC protein profile (Fig. 3d). At 48 h, both in GMAZA and DMAZA MyHC content was already higher compared with control (Fig. 3d, FC: $1.60 \pm 0.03$ GMAZA vs. GM, FC: $3.28 \pm 0.03$ DMAZA vs. $\mathrm{GM}, \mathrm{F}=94.05, p \leq 0.00001$ anova test followed by Bonferroni post hoc test). In GMAZA, extent of MyHC increase was less than in DMAZA but was prolonged in time (at $72 \mathrm{~h}$ FC: $1.49 \pm 0.12$ GMAZA vs. GM, at $96 \mathrm{~h} \mathrm{FC:} 2.01 \pm 0.11$ GMAZA vs. $\mathrm{GM} ; \mathrm{F}=94.05, p \leq 0.00001$ anova test followed by Bonferroni post hoc test). As expected, in DM condition MyHC amount increased only at $72 \mathrm{~h}$ (Fig. 3d FC: $2.56 \pm 0.17 \mathrm{DM}$ vs. GM).

These results suggest that AZA has a positive role in MyHC expression and, in association with DM, this effectiveness AZA action is accelerated.

To verify the importance of DNA demethylation during differentiation, we performed morphological analysis, immunostaining $\mathrm{C} 2 \mathrm{C} 12$ cells with MyHC antibody.

At $48 \mathrm{~h}$ (Fig. 4a) in GM condition, MyHC positive cells had small dimensions and shape slightly elongated. 
Fig. 3 Immunoblotting analysis. a Representative immunoblots of analyzed proteins are shown. MyoD (b), Myf6 (c), and MyHC (d) proteins expression was detected by western blot analysis after 48, 72, and $96 \mathrm{~h}$ of differentiation induction. $\mathrm{C} 2 \mathrm{C} 12$ myoblasts cultured in four different conditions (GM, GMAZA, DM, DMAZA). Data, obtained from three independent experiments, are expressed as fold changes (FC) relative to GM condition. The average, expressed as fold changes (FC) relative to control, are graphically represented. Data were obtained from three independent experiments. Comparisons were performed using one-way analysis of variance (ANOVA) followed by Bonferroni post hoc tests $(* * * * P<0.00001)$

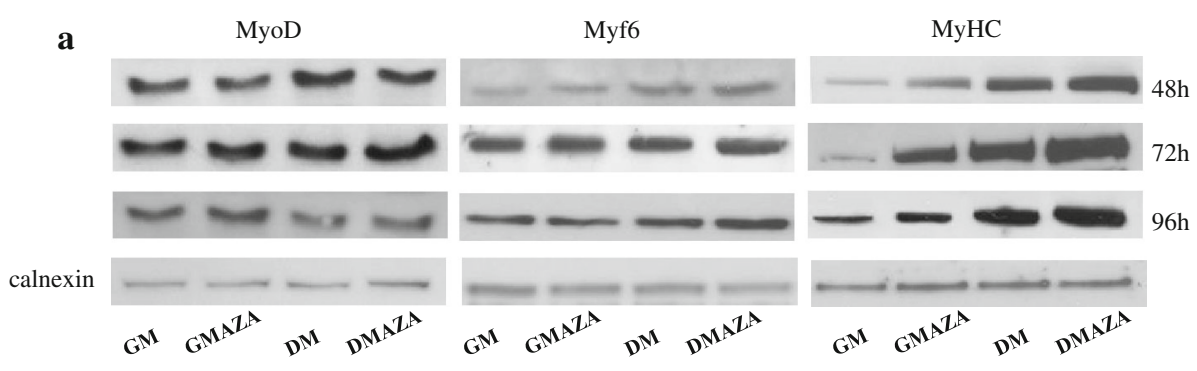

b $\quad 2,0] \quad$ MyoD protein content

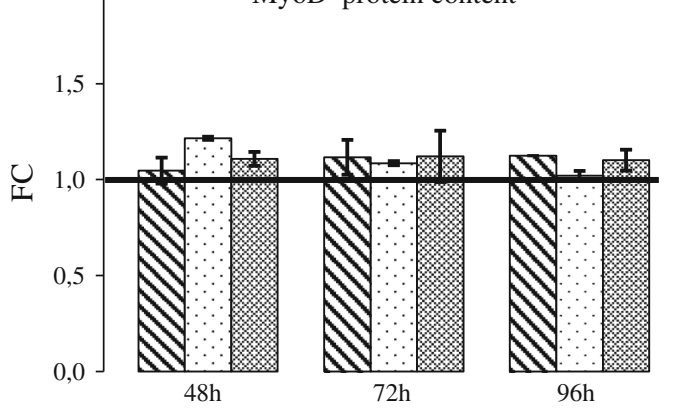

c 4,0$] \quad$ Myf6 protein content
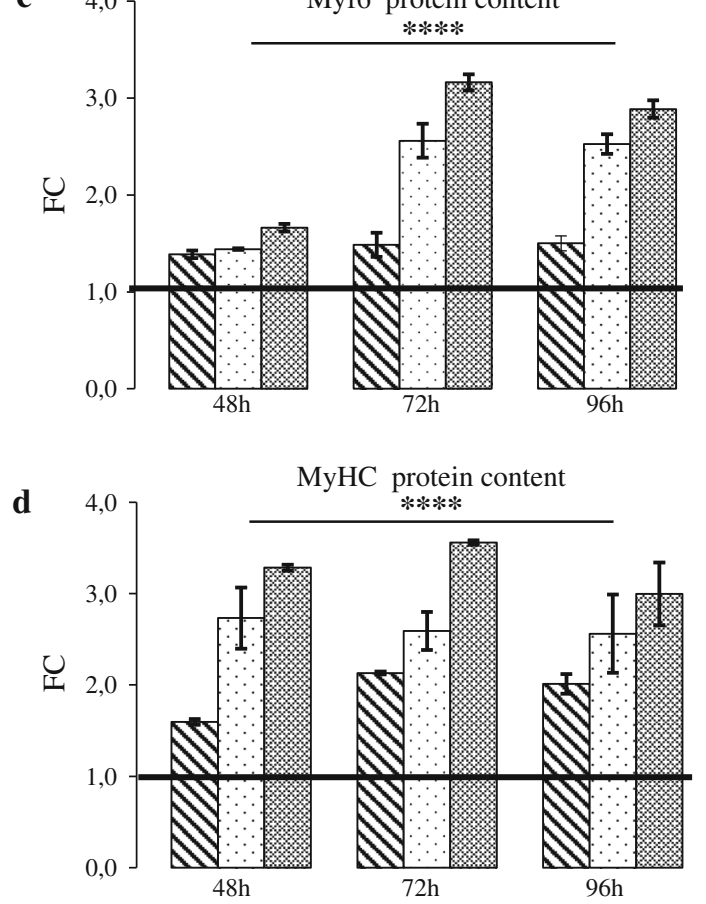

GM $\bigcirc$ GMAZA $\because$ DM ॠMAZA
GMAZA cells were more elongated, assumed a bipolar morphology and started to orient themselves in the same direction. In DM cells, these processes were more advanced and some groups of cells started to fuse. In DMAZA, this aggregation state was more evident and numerous myotubes were formed. At 72 h, GM cells fused into clusters, while in DMAZA condition organized myotubes were observed. GMAZA and DM cells were in an intermediate condition and showed morphology very similar to each other (Fig. 4b). At 96 h (Fig. 4c), already in GM condition, neo formed myotubes were observed. GMAZA and DM cells had similar morphology, suggesting that AZA action on $\mathrm{C} 2 \mathrm{C} 12$ myoblasts is superimposable to DM effect. DMAZA myotubes appeared hypertrophic and these morphological features suggest that DNA hypomethylation could improve myotube formation efficiency. 
Fig. 4 Myotubes

immunofluorescence. Analysis and morphological study. MyHC immunofluorescence analysis of $\mathrm{C} 2 \mathrm{C} 12$ cells, differentiated for 48 (a), 72 (b), and 96 (c) hours in GM, GMAZA, DM, and DMAZA conditions. MyHC positive cells are labeled in red.

Representative DAPI images are reported. The length $(\mathbf{d})$ and diameter (e) of MyHC positive myotubes were measured. The average, expressed as fold changes (FC) relative to GM control, are graphically represented. Data were obtained from three independent experiments. Comparisons were performed using one-way analysis of variance (ANOVA) followed by Bonferroni post hoc tests $(* * * * P<0.00001)$

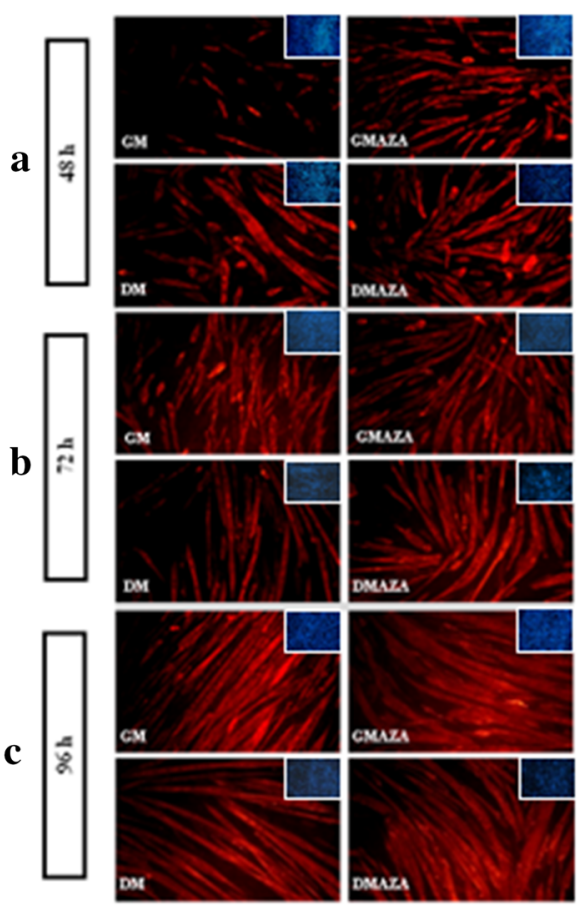

Myotubes length

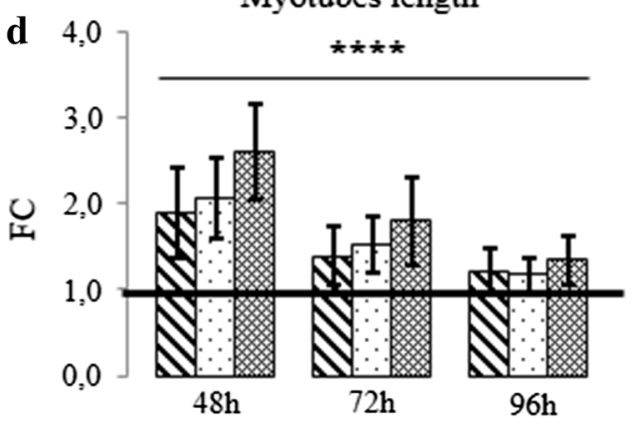

Myotubes diameter

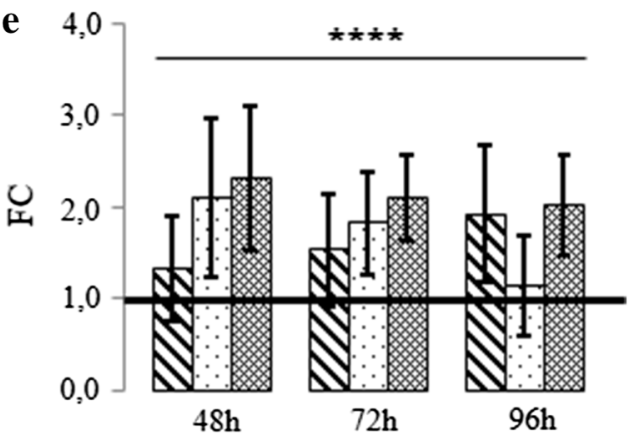

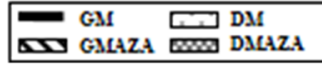

To support AZA involvement in muscle hypertrophy, myotubes dimensions were measured. During all phases, GMAZA and DMAZA myotubes showed a significant increase in diameter and length compared to GM (Fig. 4, at 48 h diameter FC: $1.33 \pm 0.58$ GMAZA vs. GM, $2.32 \pm 0.79$ DMAZA vs. GM; at $48 \mathrm{~h}$ length FC: $1.90 \pm 0.52$ GMAZA vs. GM, $2.60 \pm 0.56$ DMAZA vs. GM; at 72 h diameter FC: $1.53 \pm 0.61$ GMAZA vs. GM, $2.10 \pm 0.47$ DMAZA vs. $\mathrm{GM}$; at $72 \mathrm{~h}$ length FC: $1.40 \pm 0.34$ GMAZA vs. GM, $1.80 \pm 0.52$ DMAZA vs. $\mathrm{GM}$; at $96 \mathrm{~h}$ diameter FC: $1.93 \pm 0.75$ GMAZA vs. GM, $2.03 \pm 0.54$ DMAZA vs. GM; at $96 \mathrm{~h}$ length FC: $1.21 \pm 0.27$ GMAZA vs. GM, $1.34 \pm 0.28$ DMAZA vs. GM; for length $\mathrm{F}=17.61$, $p \leq 0.00001$, for diameter $\mathrm{F}=27.24, p \leq 0.00001$ anova test followed by Bonferroni post hoc test). Moreover, at $96 \mathrm{~h}$, there was still a significant difference between the diameter of GMAZA myotubes and those kept in GM, which instead disappears between the diameters of myotubes grown in DM compared to GM, suggesting a hypertrophic effect of AZA.

Furthermore, we studied myotubes nuclear disposition during late phase of myogenic differentiation $(96 \mathrm{~h})$. As shown in Fig. 5, immunofluorescence analysis, using antibodies against p21 and Myostatin, revealed that AZA treated myotubes were characterized by a particular arrangement of nuclei to form a ring. This nuclear organization represents a morphological marker of in vitro muscle hypertrophy [47].
To confirm DNA demethylation influence in hypertrophic process, we investigated AZA action on signaling pathway involved in hypertrophy using $\mathrm{C} 2 \mathrm{C} 12$ neo formed myotubes. C2C12 neo formed myotubes, after $72 \mathrm{~h}$ of differentiation, were treated with AZA for $24 \mathrm{~h}$ (AZAMT). Control cells were maintained in DM medium (DMMT), Fig. 6a. As expected, 5-mC ELISA assay revealed a significant DNA hypomethylation in AZAMT compared with unstimulated neo myotubes (Fig. 6b, FC: $0.23 \pm 0.02$ AZAMT vs. DMMT, $p \leq 0.01$ ).

Insulin growth factor I represents a crucial factor in skeletal muscle mass regulation and its activity predominantly requires the binding to IGF-I R [10, 24]. IGF-I R is synthesized as a single polypeptide chain (Pro IGF-I R) that is processed to mature receptor. The previous studies suggest that IGF-I promotes muscle hypertrophy by p70 S6 K and 4E-BP1 activation. 4E-BP1 phosphorylation frees eIF-4E promoting the protein synthesis beginning [24, 29, 31].

Pro IGF-I R protein level was higher in AZA treated myotubes (AZAMT) than in control (DMMT) (Fig. 6d FC: $1.66 \pm 0.03$ AZAMT vs. DMMT, $p \leq 0.02$ ), while AZA action on IGF-1 R expression was less prominent (Fig. 6d, FC: $1.32 \pm 0.04$ AZAMT vs. DMMT, $p \leq 0.05$ ). Probably, deep changes in IGF-I R synthesis could be observed after $96 \mathrm{~h}$ of myotubes differentiation.

As expected, AZA treated myotubes were associated with enhanced activation of p70 S6 K, as shown by the increase of the ratio between phosphorylated vs. un- 
a
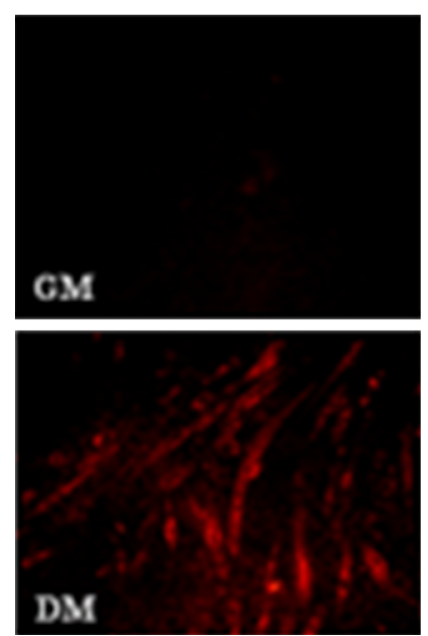
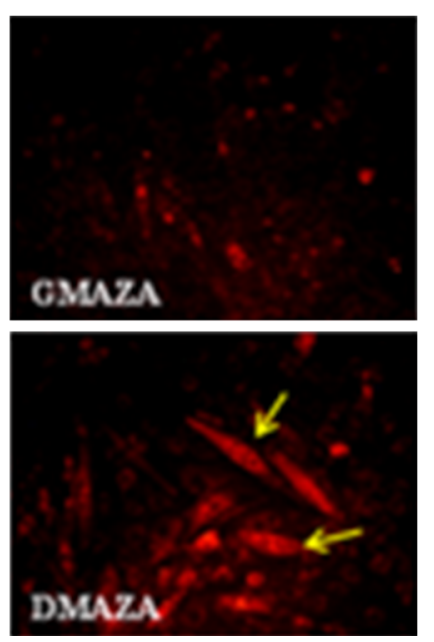

b
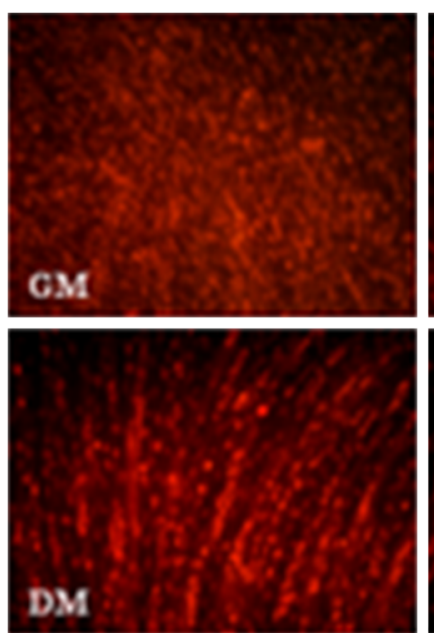
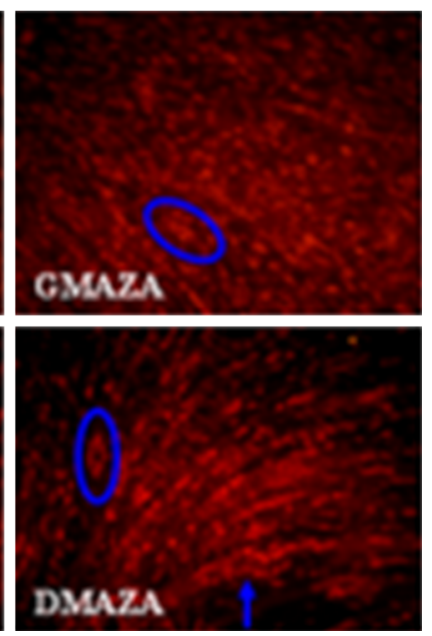

Fig. 5 Hypertrophic myotubes morphological study. p21 (a) and Mnst (b) immufluorescence analysis of C2C12 cells cultured in GM, GMAZA, DM, and DMAZA conditions after $96 \mathrm{~h}$ of differentiation. p21 and Mnst positive cells are labeled in red

phosphorylated protein (Fig. 6e, p70 S6 K/p70 S6 K FC: $1.36 \pm 0.03$ AZAMT vs. DMMT, $p \leq 0.04)$.

4E-BP1 has three electrophoretic forms, designated $\alpha, \beta$, and $\gamma$ in order of increasing mobility, inversely related to their phosphorylation state [31]. In our myotubes, 4E-BP1 was present only in the $\alpha$ and $\beta$ forms. AZA stimuli decreased the amount of $\alpha$ form and increased $\beta$ form content, as evidenced by the two isoforms ratio (4E-BP1 $\beta$ / 4E-BP1 $\alpha$ ) (Fig. 6f, FC: $1.50 \pm 0.06$ AZAMT vs. DMMT, $p \leq 0.05$ ). Moreover, we observed an eIF-4E protein level increment in AZA neo formed myotubes (Fig. 6g, FC: $1.31 \pm 0.03$ AZAMT vs. DMMT, $p \leq 0.04$ ).

Insulin growth factor I signaling pathway actives the MAPK pathway. ERK1 and ERK2, principal members of MAPK cascade, play a key role in hypertrophic process [10, 26]. We studied ERK 1 and ERK2 activation: AZA treatment increased the ratio between phosphorylated versus un-phosphorylated ERK1 and ERK2 (Fig. 6h, i, pERK1/ERK1 FC: $1.74 \pm 0.02$ AZAMT vs. DMMT, $p \leq 0.04$, Fig. 5i, pERK2/ ERK2 FC: $1.55 \pm 0.05$ AZAMT vs. DMMT, $p \leq 0.05)$.

Furthermore, Fig. 6j showed an increased tendency in MyHC protein amount in AZA treated myotubes, suggesting an activation of the protein translational machinery (FC: $1.21 \pm 0.02$ AZAMT vs. DMMT, $p \leq 0.00001$ ) and the signaling cascades involved in hypertrophic mechanisms. Finally, to confirm this deduction bright field microscopy images (Fig. 6k) indicated that in AZAMT cell the hypertrophic process was more marked.

\section{Discussion}

Several investigators [34, 40, 45, 48], including us, have shown that DNA demethylation promote MRFs expression and skeletal muscle differentiation induction. In the present study we explored AZA treatment effects during myoblasts differentiation. We, also, investigated AZA action on neo formed myotubes to evaluate its possible impact on hypertrophic process.

Our results demonstrated that during intermediate and late differentiation phase AZA strengthens and promotes Myf6 and MyHC expression compared to untreated cells. The morphological analysis confirmed that myoblasts aggregation, orientation, and fusion come earlier in AZA treated cells. Also, AZA stimuli increases myotubes length and diameter. Therefore, AZA demethylating action shortens the differentiation process, maintaining the ordered and sequential expression of the muscle-specific gene program.

The present in vitro data lead to a number of considerations.

First, our findings point to the interactions between AZA and DM on muscle cells differentiation. Specifically, the AZA-DM association has a synergistic action in enhancing myogenic process and giving hypertrophic stimuli. In fact, only in DMAZA condition p21 and Mnst-positive myotubes showed the nuclear distribution shaped a ring, typical of muscle hypertrophy in vitro [47].

Second, we investigated AZA action on IGF-I signaling pathway involved in skeletal muscle hypertrophy. We observed that AZA-induced DNA hypomethylation could improve IGF-I R protein synthesis. This is supported by a recent study demonstrating that IGF-I R synthesis is regulated by DNA methylation [49]. We evaluated the AZA action on p70 S6 K, 4E-BP1, and eIF-4E proteins, essential regulators of protein synthesis. We demonstrated that AZA activates these proteins that, at the end, lead to a raise of MyHC protein content, reflecting the increased overall 

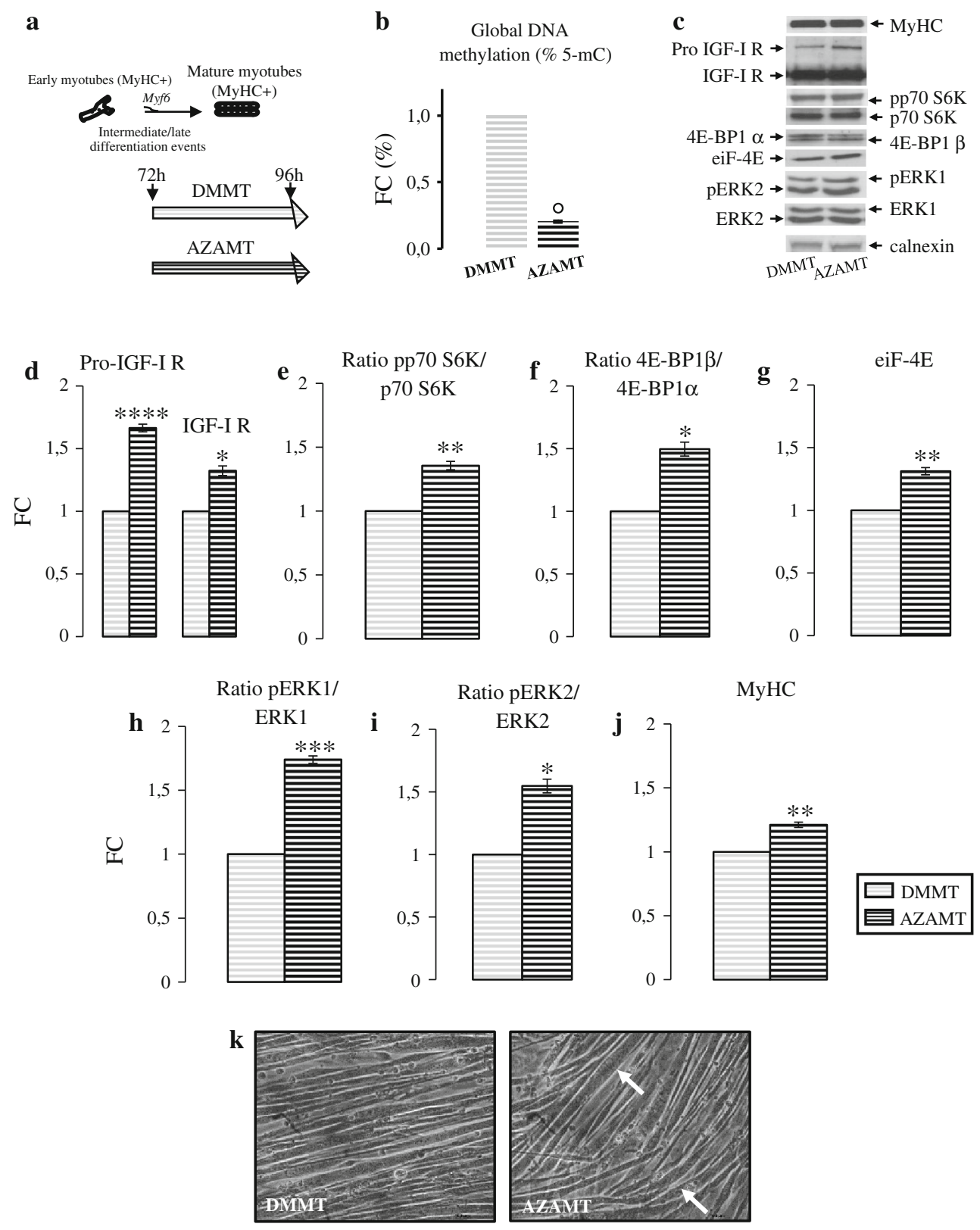

Fig. 6 Insulin growth factor I signaling pathway study. a Simplified scheme of experimental protocol: $\mathrm{C} 2 \mathrm{C} 12$ cells, after $72 \mathrm{~h}$ of differentiation induction, were treated for $24 \mathrm{~h}$ with (AZAMT) or without (DMMT) AZA. b Evaluation of AZA demethylation action on global DNA methylation status. Global DNA methylation levels were evaluated by an ELISA assay specific for 5-mC. c Representative immunoblots of analyzed proteins are shown: western blot analysis of

protein synthesis. These results suggest the possible correlation between epigenetic mechanisms and muscle protein accretion.

Importantly, our data may have a potential impact in vivo regulation of protein metabolism. In fact, IGF-I is a fundamental mediator of the hormonal and metabolic action of Growth Hormone. GH is secreted in many

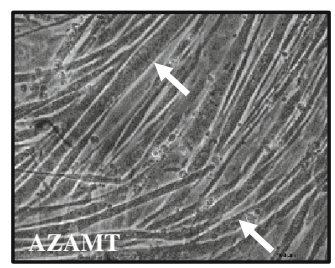

Pro IGF-I R and IGF-I R (d), pp70 S6 K/p70 S6 K ratio (e), 4EBP1 $\beta / 4 \mathrm{E}-\mathrm{BP} 1 \alpha$ ratio (f), eiF-4E (g), pERK1/ERK1 ratio (h), pERK2/ ERK2 ratio (i), and MyHC (j). Images obtained by bright field microscopy at the end of experiment (k). Data, obtained from three independent experiments, are expressed as mean \pm SD fold changes (FC) relative to DMMT. Significance: $* p<0.05, * * p<0.04$, $* * * p<0.03, * * * * p<0.02,{ }^{\circ} p<0.00001$ versus DMMT condition

physiological and stress-related conditions [50]. Our results suggest that AZA might be synergistic to GH in fostering the IGF-I mediated actions [51]. We may speculate an hypothetical clinical use of demethylating agents in conditions of muscle mass impairment/hypotrophy. A synergistic effect of 5-azacytidine and hGH-V (human placental growth hormone) was previously established [52]. 
Third, the demonstration that AZA-induced DNA demethylation accelerates the skeletal muscle differentiation might constitute a new model to study the pathophysiology of several genetic muscle diseases. Recent studies have evidenced that DNA hypermethylation is correlated with genetic skeletal muscle diseases, like Duchenne muscular and myotonic dystrophy [53, 54]. Hupkes et al. [40] have reported that AZA induced-DNA demethylation enhances skeletal muscle function, in particular AZA promotes the acquisition of spontaneous contractility in $\mathrm{C} 2 \mathrm{C} 12$ cell model. Accordingly and concordant with these data, our results demonstrate that DNA hypomethylation could improve skeletal muscle differentiation. Taken together, these data confirm the idea that the DNA methylation could represents the epigenetic key point regulating skeletal muscle differentiation and that drugs or other stimuli, which promote DNA demethylation, could modulate muscle differentiation.

Fourth, our observation that AZA and DM association induces hypertrophic response implies a link with the most important physiological stimulus leading to muscle hypertrophy: exercise training. Very recently, Barrès et al. [55] demonstrated that acute exercise leads to transient changes in DNA methylation in key genes of skeletal muscle metabolism. In addition, our previous study that showed a significant association between elité athletes phenotype (enhanced muscle mass) and polymorphic variants involved in DNA methylation [48]. Considering all together, the above considerations suggest an important regulatory role of DNA methylation on muscle mass accretion.

In conclusion, we demonstrated that DNA demethylation enhances myoblasts differentiation and hypertrophy during the late phase of myogenesis and that this effect is mediated via activation of the IGF-I receptor and postreceptor phosphorylation cascade. The present results constitute a proof of principle of potential effects of 5 -azacytidine on muscle protein accretion.

Conflict of interest The authors declare that they have no conflict of interest.

Open Access This article is distributed under the terms of the Creative Commons Attribution License which permits any use, distribution, and reproduction in any medium, provided the original author(s) and the source are credited.

\section{References}

1. R. Bassel-Duby, E.N. Olson, Signaling pathways in skeletal muscle remodeling. Annu. Rev. Biochem. 75, 19-37 (2006)

2. G. Cossu, S. Biressi, Satellite cells, myoblasts and other occasional myogenic progenitors: possible origin, phenotypic features and role in muscle regeneration. Semin. Cell Dev. Biol. 1, 154 (2006)
3. S.B. Chargé, M.A. Rudnicki, Cellular and molecular regulation of muscle regeneration. Physiol. Rev. 84, 209-238 (2004)

4. J. Dhawan, T.A. Rando, Stem cells in postnatal myogenesis: molecular mechanisms of satellite cell quiescence, activation and replenishment. Trends Cell Biol. 12, 666-673 (2005)

5. R.L. Perry, M.A. Rudnicki, Molecular mechanisms regulating myogenic determination and differentiation. Front Biosci. 5, D750-D767 (2000)

6. O. Ostrovsky, E. Bengal, The mitogen-activated protein kinase cascade promotes myoblast cell survival by stabilizing the cyclindependent kinase inhibitor, p21WAF1 protein. J. Biol. Chem. 278, 21221-21231 (2003)

7. A.C. McPherron, A.M. Lawler, S.J. Lee, Regulation of skeletal muscle mass in mice by a new TGF $\beta$ superfamily member. Nature 387, 83-90 (1997)

8. M.S. Williams, Myostatin mutation associated with gross muscle hypertrophy in a child. N. Engl. J. Med. 351, 1030-1031 (2004)

9. W. Taylor, K. Ma, N.F. Gonzalez-Cadavid, J.N. Artaza, S. Bhasin, C. Mallidis, Endogenous expression and localization of myostatin and its relation to myosin heavy chain distribution in C2C12 skeletal muscle cells. J. Cell Physiol. 190(2), 170-179 (2002)

10. D.J. Glass, Signalling pathways that mediate skeletal muscle hypertrophy and atrophy. Nat. Cell Biol. 2, 87-90 (2003)

11. H. Kim, E. Barton, N. Muja, S. Yakar, P. Pennisi, D. Leroith, Intact insulin and insulin-like growth factor-I receptor signaling is required for growth hormone effects on skeletal muscle growth and function in vivo. Endocrinology 146, 1772-1779 (2005)

12. D.L. DeVol, P. Rotwein, J.L. Sadow, J. Novakofski, P.J. Bechtel, Activation of insulin-like growth factor gene expression during work-induced skeletal muscle growth. Am. J. Physiol. 259, E89E95 (1990)

13. E.E. Spangenburg, D. Le Roith, C.W. Ward, S.C. Bodine, A functional insulin-like growth factor receptor is not necessary for load induced skeletal muscle hypertrophy. J. Physiol. 586, 283-291 (2008)

14. A.M. Fernandez, J. Dupont, R.P. Farrar, S. Lee, B. Stannard, D. Le Roith, Muscle-specific inactivation of the IGF-I receptor induces compensatory hyperplasia in skeletal muscle. J. Clin. Invest. 109, 347-355 (2002)

15. M. Miyazaki, J.J. McCarthy, M.J. Fedele, K.A. Esser, Early activation of mTORC1 signalling in response to mechanical overload is independent of phosphoinositide 3-kinase/Akt signalling. J. Physiol. 589, 1831-1846 (2011)

16. K.A. Esser, J.J. McCarthy, M. Miyazaki, Comments on point: counterpoint: IGF is/is not the major physiological regulator of muscle mass. IGF-1 is not key for adult skeletal muscle hypertrophy. J. Appl. Physiol. 108, 1830 (2010)

17. M. Vinciguerra, M. Hede, N. Rosenthal, Comments on point: counterpoint: IGF is/is not the major physiological regulator of muscle mass. IGF-1 is a major regulator of muscle mass during growth but not for adult myofiber hypertrophy. J. Appl. Physiol. 108, 1829-1830 (2010)

18. T. Shavlakadze, M.D. Grounds, Comments on point: counterpoint: IGF is/is not the major physiological regulator of muscle mass. IGF-1 is a major regulator of muscle mass during growth but not for adult myofiber hypertrophy. J. Appl. Physiol. 108, 1829 (2010)

19. E.E. Spangenburg, Comments on point: counterpoint: IGF is/is not the major physiological regulator of muscle mass. hypertrophy without IGF-I? J. Appl. Physiol. 108, 1825 (2010)

20. A. Musarò, K. McCullagh, A. Paul, L. Houghton, G. Dobrowolny, M. Molinaro, E.R. Barton, H.L. Sweeney, N. Rosenthal, Localized Igf-1 transgene expression sustains hypertrophy and regeneration in senescent skeletal muscle. Nat. Genet. 27, 195-200 (2001) 
21. A. Musarò, N. Rosenthal, The critical role of insulin-like growth factor-1 isoforms in the physiopathology of skeletal muscle. Curr. Genomics 3, 19-32 (2006)

22. M. Sandri, C. Sandri, A. Gilbert, C. Skurk, E. Calabria, A. Picard, K. Walsh, S. Schiaffino, S.H. Lecker, A.L. Goldberg, Foxo transcription factors induce the atrophy-related ubiquitin ligase atrogin-1 and cause skeletal muscle atrophy. Cell 117, 399-412 (2004)

23. J.P. Liu, J. Baker, A.S. Perkins, E.J. Robertson, A. Efstratiadis, Mice carrying null mutations of the genes encoding insulin-like growth factor I (Igf-1) and type 1 IGF receptor (Igf1r). Cell 75, 59-72 (1993)

24. C. Rommel, S.C. Bodine, B.A. Clarke, R. Rossman, L. Nunez, T.N. Stitt, G.D. Yancopoulos, D.J. Glass, Mediation of IGF-1induced skeletal myotube hypertrophy by $\mathrm{PI}(3) \mathrm{K} / \mathrm{Akt} / \mathrm{mTOR}$ and PI(3)K/Akt/GSK3 pathways. Nat. Cell Biol. 3, 1009-1013 (2001)

25. A. Plotnikov, E. Zehorai, S. Procaccia, R. Seger, The MAPK cascades: signaling components, nuclear roles and mechanisms of nuclear translocation. Biochem. Biophys. Acta. 1813, 1619-1633 (2011)

26. J.D. Knight, R. Kothary, The myogenic kinome: protein kinases critical to mammalian skeletal myogenesis. Skelet. Muscle 1, 29 (2011)

27. J. Li, S.E. Johnson, ERK2 is required for efficient terminal differentiation of skeletal myoblasts. Biochem. Biophys. Res. Commun. 345(4), 1425-1433 (2006)

28. A.J. Galpin, A.C. Fry, L.Z. Chiu, D.B. Thomason, B.K. Schilling, High-power resistance exercise induces MAPK phosphorylation in weightlifting trained men. Appl. Physiol. Nutr. Metab. 37, 80-87 (2012)

29. C.H. Lee, K. Inoki, K.L. Guan, mTOR pathway as a target in tissue hypertrophy. Annu. Rev. Pharmacol. Toxicol. 47, 443-467 (2007)

30. S. Fujita, T. Abe, M.J. Drummond, J.G. Cadenas, H.C. Dreyer, Y. Sato, E. Volpi, B.B. Rasmussen, Blood flow restriction during low-intensity resistance exercise increases S6K1 phosphorylation and muscle protein synthesis. J. Appl. Physiol. 103, 903-910 (2007)

31. T.A. Haystead, C.M. Haystead, C. Hu, T.A. Lin, J.C.J.R. Lawrence, Phosphorylation of PHAS-I by mitogen-activated protein (MAP) kinase. identification of a site phosphorylated by MAP kinase in vitro and in response to insulin in rat adipocytes. J. Biol. Chem. 269, 23185-23191 (1994)

32. N. Kubica, S.R. Kimball, L.S. Jefferson, P.A. Farrell, Alterations in the expression of mRNAs and proteins that code for species relevant to eIf2B activity after an acute bout of resistance exercise. J. Appl. Physiol. 96, 679-687 (2004)

33. D. Palacios, P.L. Puri, The epigenetic network regulating muscle development and regeneration. J. Cell Physiol. 207, 1-11 (2006)

34. S.M. Taylor, P.A. Jones, Changes in phenotypic expression in embryonic and adult cells treated with 5-azacytidine. J. Cell Physiol. 111, 187-194 (1982)

35. B.P. Brunk, D.J. Goldhamer, C.P. Emerson, Regulated demethylation of the myoD distal enhancer during skeletal myogenesis. Dev. Biol. 177, 490-503 (1996)

36. M. Lucarelli, A. Fuso, R. Strom, S. Scarpa, The dynamics of myogenin site-specific demethylation is strongly correlated with its expression and with muscle differentiation. J. Biol. Chem. 276, 7500-7506 (2001)

37. J.K. Christman, 5-Azacytidine and 5-aza-2'-deoxycytidine as inhibitors of DNA methylation: mechanistic studies and their implications for cancer therapy. Oncogene 21, 5483-5495 (2002)

38. S. Burattini, P. Ferri, M. Battistelli, R. Curci, F. Luchetti, E. Falcieri, C2C12 murine myoblasts as a model of skeletal muscle development: a morphofunctional characterization. Eur. J. Histochem. 48, 223-233 (2004)

39. G. Cossu, R. Kelly, S. Di Donna, E. Vivarelli, M. Buckingham, Myoblast differentiation during mammalian somitogenesis is dependent upon a community effect. Proc. Natl. Acad. Sci. USA 92(6), 2254-2258 (1995)

40. M. Hupkes, M.K. Jonsson, W.J. Scheenen, W. van Rotterdam, A.M. Sotoca, E.P. van Someren, M.A. van der Heyden, T.A. van Veen, R.I. van Ravestein-van Os, S. Bauerschmidt, E. Piek, D.L. Ypey, E.J. van Zoelen, K.J. Dechering, Epigenetics: DNA demethylation promotes skeletal myotube maturation. FASEB J. 25, 3861-3872 (2011)

41. P. Senesi, L. Luzi, A. Montesano, N. Mazzocchi, I. Terruzzi, Betaine supplement enhances skeletal muscle differentiation in murine myoblasts via IGF-1 signaling activation. J. Transl. Med. 11(1), 174 (2013)

42. S. Fernàndez-Roig, S.C. Lai, M.M. Murphy, J. Fernandez-Ballart, E.V. Quadros, Vitamin B12 deficiency in the brain leads to DNA hypomethylation in the TCblR/CD320 knockout mouse. Nutr. Metab. (Lond.) 9, 41 (2012)

43. I. Terruzzi, P. Senesi, C. Magni, A. Montesano, A. Scarafoni, L. Luzi, M. Duranti, Insulin-mimetic action of conglutin- $\gamma$, a lupin seed protein, in mouse myoblasts. Nutr. Metab. Cardiovasc. Dis. 21, 197-205 (2011)

44. M. Menconi, P. Gonnella, V. Petkova, S. Lecker, P.O. Hasselgren, Dexamethasone and corticosterone induce similar, but not identical, muscle wasting responses in cultured L6 and $\mathrm{C} 2 \mathrm{C} 12$ myotubes. J. Cell. Biochem. 105(2), 353-364 (2008)

45. A. Montesano, 1 Luzi, P. Senesi, I. Terruzzi, Modulation of cell cycle progression by 5-azacytidine is associated with early myogenesis induction in murine myoblasts. Int. J. Biol. Sci. 9(4), 391-402 (2013)

46. P. Ferri, E. Barbieri, S. Burattini, M. Guescini, A. D’Emilio, L. Biagiotti, P. Del Grande, A. De Luca, V. Stocchi, E. Falcieri, Expression and subcellular localization of myogenic regulatory factors during the differentiation of skeletal muscle $\mathrm{C} 2 \mathrm{C} 12$ myoblasts. J. Cell Biochem. 108, 1302-1317 (2009)

47. A. Musarò, N. Rosenthal, Maturation of the myogenic program is induced by postmitotic expression of insulin-like growth factor I. Mol. Cell Biol. 4, 3115-3124 (1999)

48. I. Terruzzi, P. Senesi, A. Montesano, A. La Torre, G. Alberti, S. Benedini, L. Luzi, Genetic polymorphisms of the enzymes involved in DNA methylation and synthesis in elite athletes. Physiol. Genomics 43, 965-973 (2011)

49. A. Nikoshkov, V. Sunkari, O. Savu, E. Forsberg, S.B. Catrina, K. Brismar, Epigenetic DNA methylation in the promoters of the Igf1 receptor and insulin receptor genes in $\mathrm{db} / \mathrm{db}$ mice. Epigenetics 4, 405-409 (2011)

50. I.E. Elijah, L.K. Branski, C.C. Finnerty, D.N. Herndon, The GH/ IGF-1 system in critical illness. Best Pract. Res. Clin. Endocrinol. Metab. 25, 759-767 (2011)

51. D.J. Chia, P. Rotwein, Defining the epigenetic actions of growth hormone: acute chromatin changes accompany GH-activated gene transcription. Mol. Endocrinol. 24, 2038-2049 (2010)

52. M.M. De Jesús Romero-Prado, H.A. Barrera-Saldaña, J.L. Castrillo-Diez, hGH-V gene expression and promoter activity under glucose and 5-azacytidine (5azaC) effects. Gene 15, 7-15 (2010)

53. S. Acharyya, S.M. Sharma, A.S. Cheng, K.J. Ladner, W. He, W. Kline, H. Wang, M.C. Ostrowski, T.H. Huang, D.C. Guttridge, TNF inhibits notch-1 in skeletal muscle cells by Ezh2 and DNA methylation mediated repression: implications in duchenne muscular dystrophy. PLoS ONE 30, e 12479 (2010)

54. A. López Castel, M. Nakamori, S. Tomé, D. Chitayat, G. Gourdon, C.A. Thornton, C.E. Pearson, Expanded CTG repeat demarcates a boundary for abnormal $\mathrm{CpG}$ methylation in myotonic dystrophy patient tissues. Hum. Mol. Genet. 20, 1-15 (2011)

55. R. Barrès, J. Yan, B. Egan, J.T. Treebak, M. Rasmussen, T. Fritz, K. Caidahl, A. Krook, D.J. O'Gorman, J.R. Zierath, Acute exercise remodels promoter methylation in human skeletal muscle. Cell Metab. 15, 405-411 (2012) 